\title{
Review of the 2008-2009 pertussis epidemic in NSW: notifications and hospitalisations
}

\author{
Paula J. Spokes ${ }^{\mathrm{A}, \mathrm{C}}$, Helen E. Quinn ${ }^{\mathrm{B}}$ \\ and Jeremy M. McAnulty ${ }^{\mathrm{A}}$ \\ ${ }^{\mathrm{A}}$ Communicable Diseases Branch, NSW Department of Health \\ ${ }^{\mathrm{B}}$ National Centre for Immunisation Research and Surveillance, \\ The Children's Hospital at Westmead \\ ${ }^{\mathrm{C}}$ Corresponding author.Email: pspok@doh.health.nsw.gov.au
}

\begin{abstract}
In 2008 and 2009 increased numbers of pertussis notifications were reported in NSW. During the epidemic period, the pertussis notification rate was 2.7 times higher than the previous 5-year average. Rates of pertussis notifications and hospitalisations were highest among infants aged less than 1 year across all years studied. Compared to previous years, the notification rate for children aged 1-4 years increased dramatically and was particularly striking for children aged 3 years with notifications exceeding those for infants in 2009. Changes in testing practices during the epidemic period, including a significant increase in the use of polymerase chain reaction, may account for some of the relative increase in size of the 2008-2009 outbreak compared with previous outbreak years.
\end{abstract}

Pertussis is a notifiable condition under the New South Wales (NSW) Public Health Act 1991 by doctors, hospitals, laboratories, schools and child-care facilities. During 2008 and 2009, an increase in pertussis notifications was reported to the NSW Department of Health. A range of public health control measures were implemented in NSW in response to the outbreak to help protect infants at greatest risk of severe disease. This paper describes notification and hospitalisation data over the epidemic period.

Pertussis, or whooping cough, is a bacterial infection of the respiratory tract caused by Bordetella pertussis. Pertussis usually begins with symptoms similar to a cold, with a runny nose, tiredness and sometimes a mild fever. A cough then develops, usually in bouts (paroxysms), followed by a deep gasp (or whoop). Pertussis affects people of all ages, however, infants are at greatest risk of severe disease, complications, hospitalisation and death. ${ }^{1}$ Parents, siblings and close contacts are the most important sources of pertussis infection to infants. $^{2}$

Control of pertussis is problematic because of its high degree of communicability and because immunity, whether from immunisation or infection, wanes after approximately 6-10 years, resulting in renewed susceptibility to infection. In NSW, periodic epidemics of pertussis occur at intervals of 3-4 years, on a background of endemic circulation. ${ }^{3}$

The current NSW immunisation schedule for pertussis includes a primary course of diphtheria-tetanus-acellular pertussis (DTPa) vaccine at 2, 4 and 6 months of age with a booster dose at 4 years of age. Several modifications to this immunisation schedule have occurred since the vaccine's introduction. In 1997, DTPa vaccines replaced diphtheriatetanus-whole cell pertussis (DTPw) vaccines for booster doses and from 1999 for all doses. Prior to September 2003, an 18-month booster was included in the schedule, however this was removed based on evidence that three doses of acellular pertussis vaccine in the first year of life provide adequate protection until the age of 6 years. ${ }^{4}$ From the beginning of 2004, an adolescent/adult formulated booster (dTpa) replaced the use of the adult diphtheriatetanus vaccine (dT) at 15-17 years. The latter change was prompted by a shift in notifications to this age group following the introduction of the booster dose at 4-5 years in 1994. ${ }^{5}$ From 2003, dTpa was also recommended for use in adults who were in contact with young infants (e.g. new parents, childcare and healthcare staff), but was not funded under the National Immunisation Program.

The coverage of pertussis vaccination in the community at 12 and 24 months of age has substantially increased in NSW during the last decade. The Australian Bureau of Statistics (ABS) found coverage with three doses of DTPw at $12-23$ months of age was recorded at $87 \%$ and at $60 \%$ for four doses of DTPw at 24 months during $1995 .{ }^{6}$ The national immunisation coverage annual report, while using different survey methods, found that coverage had risen to $92 \%$ for three doses of DTPa at 12 months and $95 \%$ for the reduced schedule of three doses of DTPa at 24 months in March $2008 .^{7}$

Due to the abovementioned changes in pertussis vaccination within the National Immunisation Program, and changes in vaccine coverage, several cohorts of people with different vaccination histories now exist within the population. In NSW, epidemics occurred in 1993-1994, 


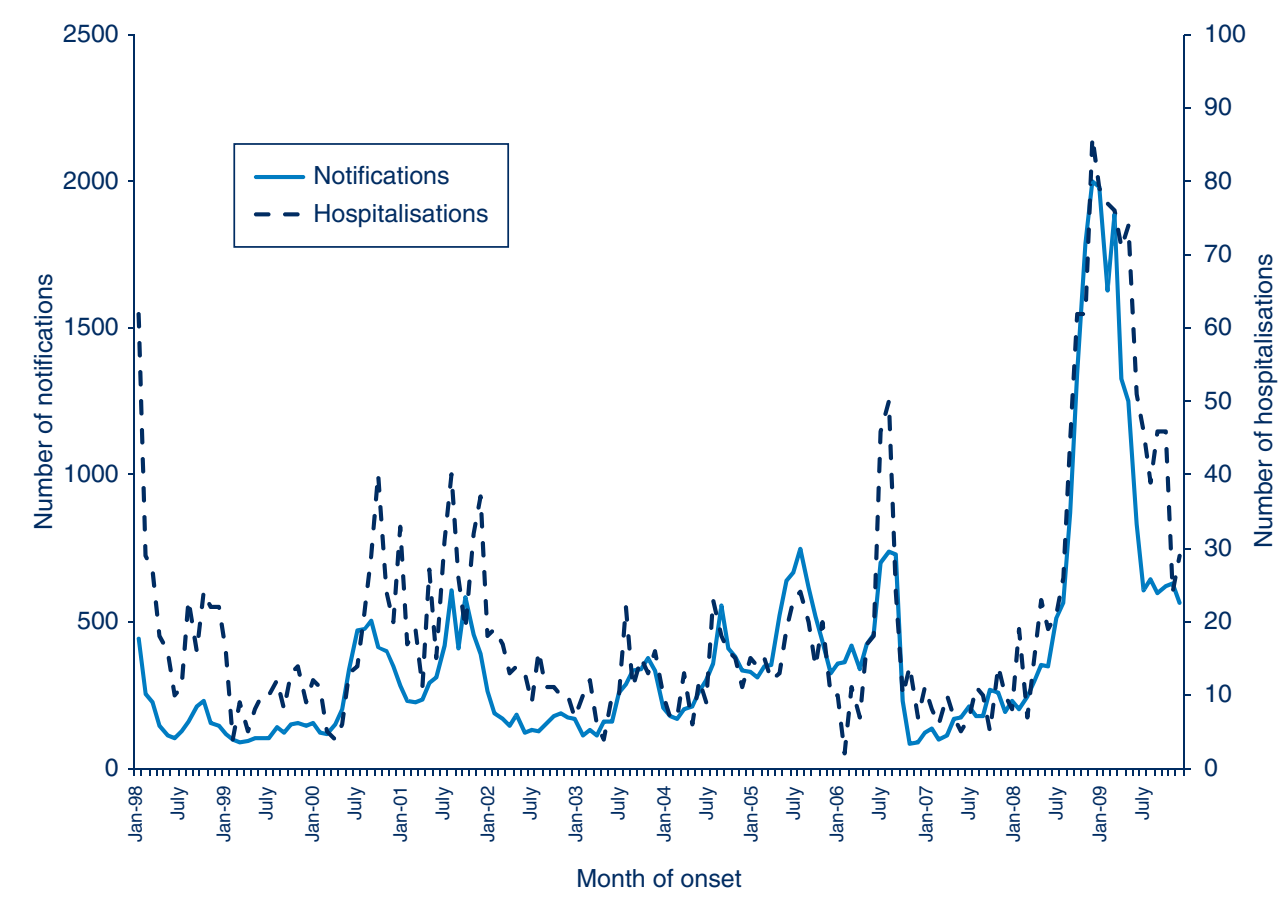

Figure 1. Number of pertussis notifications and hospitalisations by month of onset, NSW, 1998-2009.

Sources: NSW Notifiable Diseases Database and NSW Inpatient Statistics Collection, NSW Department of Health.

1997-1998 and 2000-2002, with successive upward shifts in the age distribution of cases. ${ }^{8}$ The most recent epidemic in 2005 was dominated by cases in adults whose disease was largely diagnosed by serology. ${ }^{9}$

In this paper we review the epidemiology of pertussis cases notified in NSW since 1998.

\section{Method}

\section{NSW notification data}

Pertussis notification data from the NSW Notifiable Diseases Database were reviewed for cases with a date of onset between 1 January 1998 and 31 December 2009. Vaccination status and morbidity and mortality outcomes for notified cases were obtained through follow-up by NSW public health units. All rates were calculated using ABS population estimates for the relevant year. Rates are presented as annual rates per 100000 total population or in age groups, as appropriate.

Systems for the diagnosis and counting of cases of pertussis have changed over time. From 1 January 2007, the manufacturer of a widely used commercial Bordetella IgA assay changed the recommended cut-off titres for a commonly used serological test used across NSW. From 1 January 2009, NSW ceased collecting data about suspected cases of pertussis in accordance with national surveillance protocols. ${ }^{10}$

\section{NSW hospitalisations}

Pertussis hospitalisations were obtained from the NSW Inpatients Statistics Collection with an admission date between 1 January 1998 and 31 December 2009. All rates were calculated using ABS population estimates for the relevant year. Rates are presented as annual rates per 100000 total population or population in age groups, as appropriate. Eligible hospitalisations were those with an International Classification of Diseases, tenth revision (IDC-10-AM) code of A37 (whooping cough) or a subcode, listed in the principal diagnosis or in any other diagnosis. Medians are used to describe the length of stay per admission episode as these data are not distributed normally.

\section{Results \\ Pertussis notification and hospitalisation trends}

There were 54380 notifications of pertussis with dates of onset between 1 January 1998 and 31 December 2009 (an average of 4532 notifications per year). Peaks in notifications were reported in 2001 (4439 cases; 68 per 100000 population), 2005 (5811 cases; 86 per 100000 population) and most recently in 2008 (8759 cases; 125 per 100000 population) and 2009 (12547 cases; 178 per 100000 population) (Figure 1). Notifications in the 2008-2009 epidemic period exceeded previous outbreak years and were 2.7 times higher than the previous 5-year average.

There were 2919 hospital separations coded as pertussis in NSW between 1 January 1998 and 31 December 2009 (2274 (78\%) with a principal diagnosis of pertussis). As with the notifications, periodic epidemics of pertussis can be observed. The highest numbers of annual hospitalisations occurred in 2001 (307 cases; 4.7 per 100000 population), 2006 (216 cases; 3.2 per 100000 population) and most 


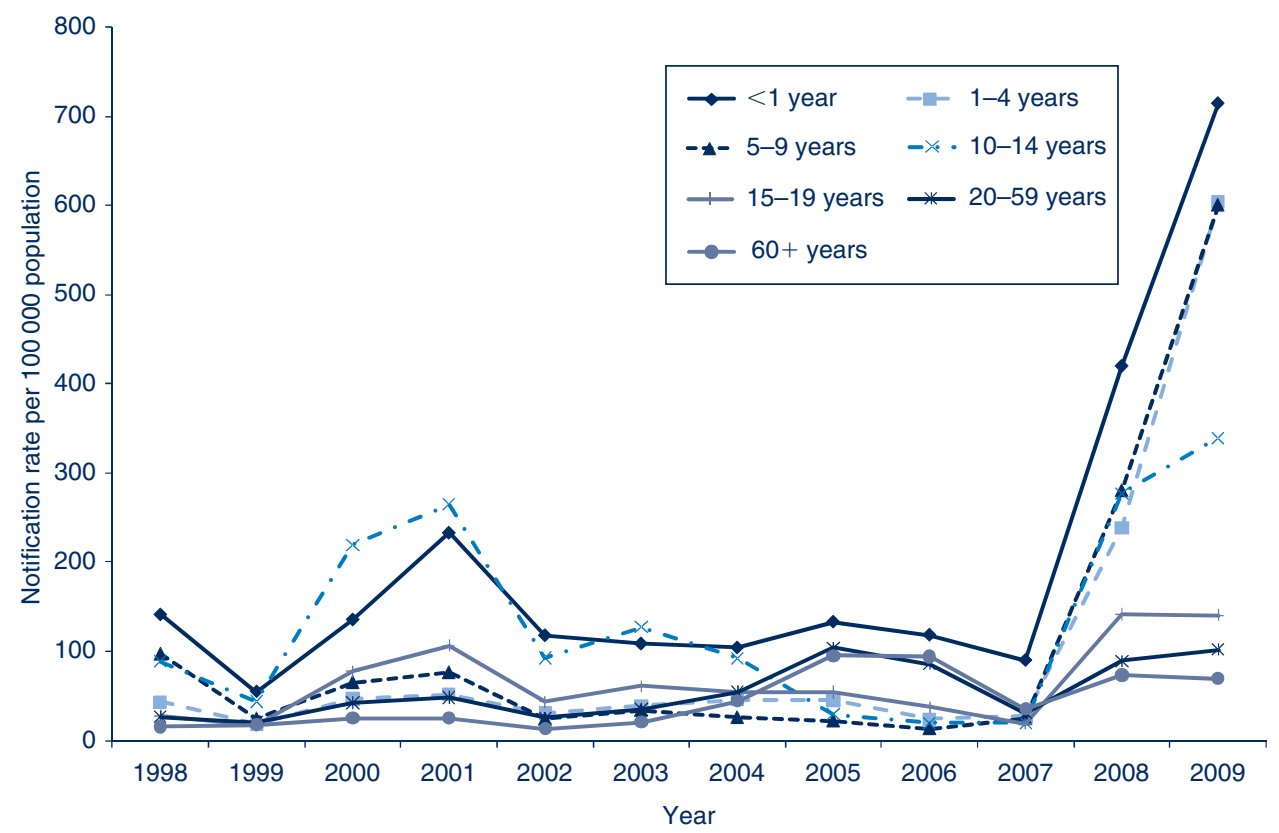

Figure 2. Age-specific pertussis notification rates by age group, NSW, 1998-2009. Sources: NSW Notifiable Diseases Database and ABS population estimates (HOIST), NSW Department of Health.

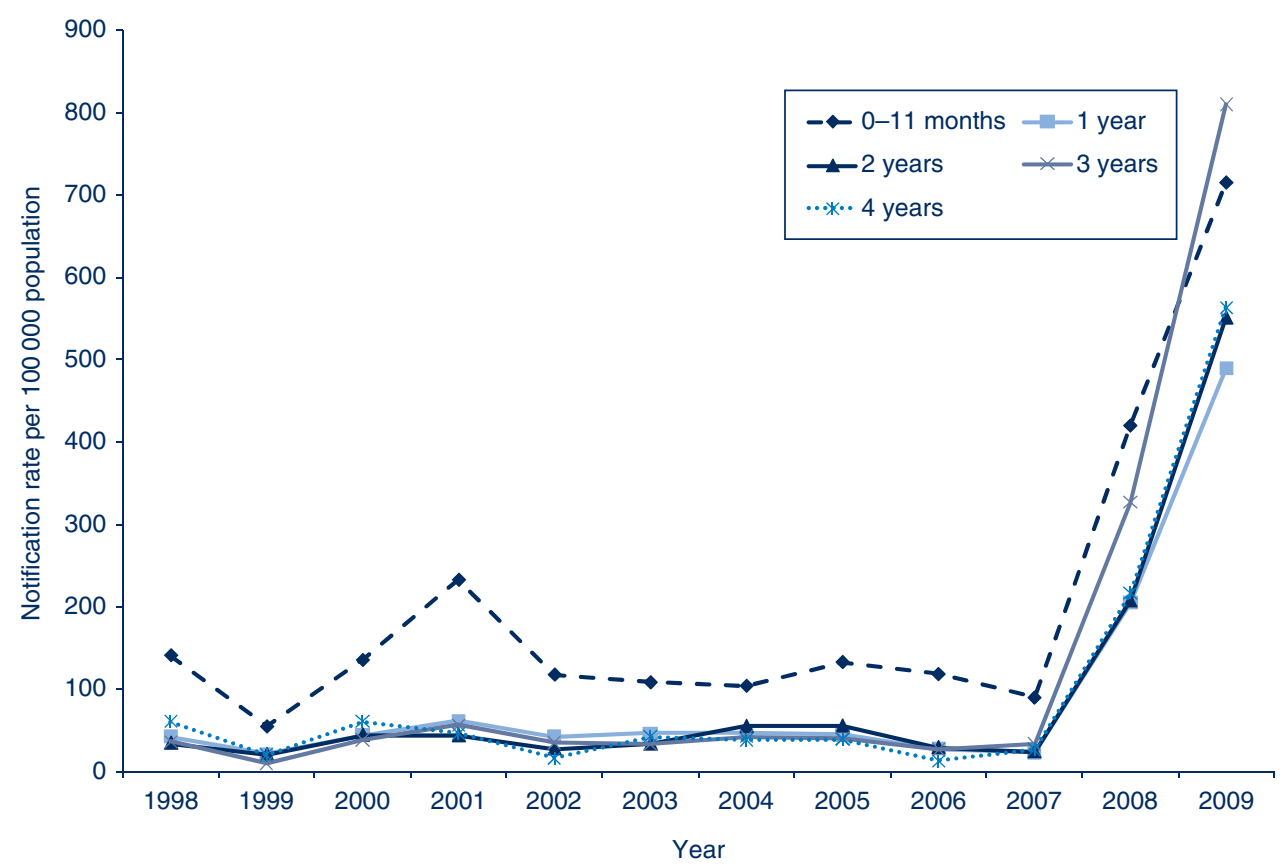

Figure 3. Age-specific pertussis notification rates in infants aged less than 5 years, NSW, 1998-2009.

Sources: NSW Notifiable Diseases Database and ABS population estimates (HOIST), NSW Department of Health.

recently in 2008 (393 cases; 5.6 per 100000 population) and 2009 (659 cases; 9.4 per 100000 population). During the 2008-2009 epidemic, the hospitalisation rate for pertussis was 3.1 times higher than the previous 5-year average.

\section{Age distribution}

Age-specific notification rates are shown in Figures 2 and 3 . The pertussis notification rate was highest among infants aged less than 1 year in the majority of years studied (annual average rate of 165 per 100000 population). In 2001, the highest age-specific notification rate was for young people aged 10-14 years (2650 per 100000 population). Notifications in young people aged 10-14 years peaked again during the 2008 (277 per 100000 population) and 2009 (339 per 100000 population) epidemic period. Notification rates for adults aged greater than 20 years 


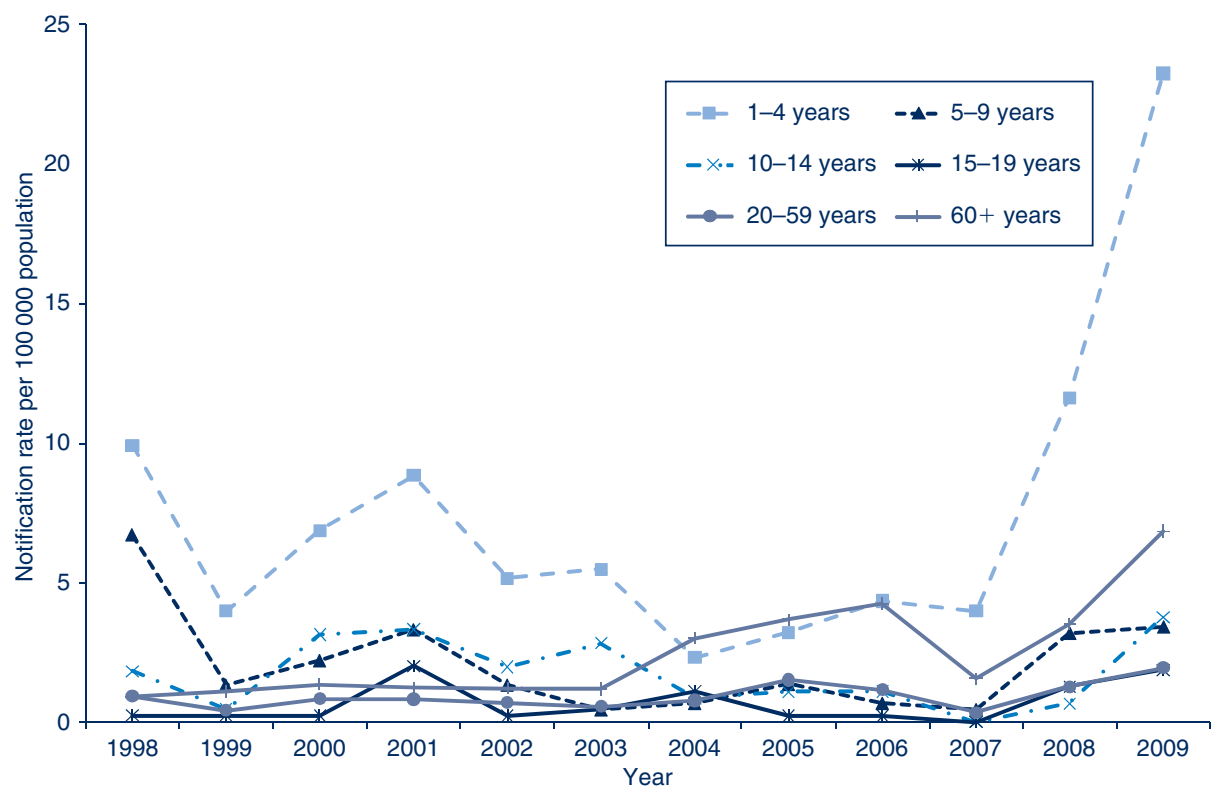

Figure 4. Age-specific pertussis hospitalisation rates, NSW, 1998-2009 by age group, excluding infants aged less than 1 year.

Sources: NSW Inpatient Statistics Collection and ABS population estimates (HOIST), NSW Department of Health.

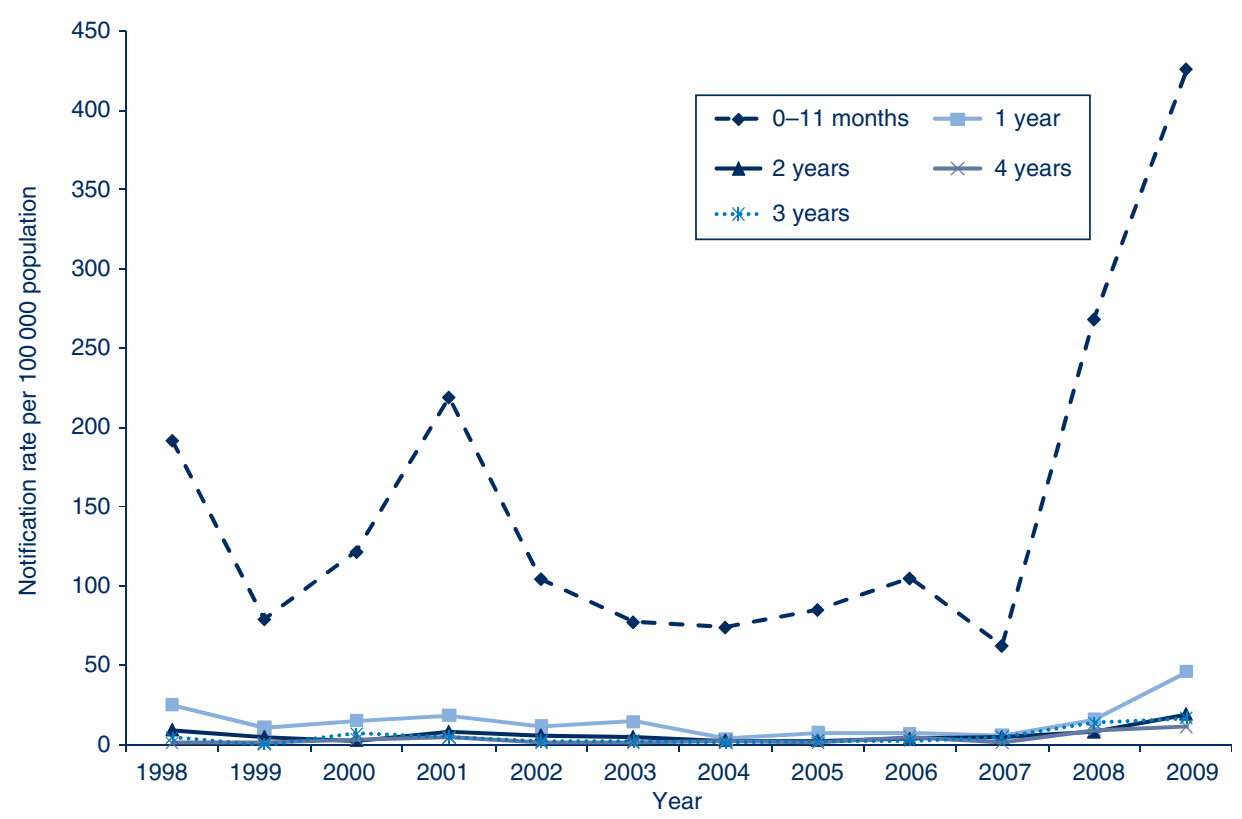

Figure 5. Pertussis hospitalisation rates in infants aged less than 5 years, NSW, 1998-2009. Sources: NSW Inpatient Statistics Collection and ABS population estimates (HOIST), NSW Department of Health.

have remained relatively stable over time with a peak reported in 2005-2006 and again during the 2008-2009 epidemic (Figure 2).

There were 4033 notifications in children aged $0-5$ years (453 per 100000 population), including 1008 aged less than 1 year (570 per 100000 population) during 2008 2009. Of the children aged less than 1 year, $41 \%$ were in infants aged less than 3 months. During the most recent epidemic in 2008-2009, the notification rate for children aged 1-4 years increased dramatically and in disproportion to patterns from previous years. This was particularly striking for children aged 3 years with notifications in this age group exceeding those in infants aged less than 1 year in 2009 (Figure 3).

The age-specific pertussis hospitalisation rates over the analysed period are shown in Figures 4 and 5. The pertussis 
Table 1. Pertussis notification and hospitalisation rates, NSW by area health service, ${ }^{\mathrm{a}} 2003-2007$ and $2008-2009$

\begin{tabular}{|c|c|c|c|c|}
\hline & $\begin{array}{l}\text { Average notification } \\
\text { rate per } 100000 \\
2003-2007\end{array}$ & $\begin{array}{l}\text { Average notification } \\
\text { rate per } 100000 \\
2008-2009\end{array}$ & $\begin{array}{l}\text { Average hospitalisation } \\
\text { rate per } 100000 \\
2003-2007\end{array}$ & $\begin{array}{l}\text { Average hospitalisation } \\
\text { rate per } 100000 \\
2008-2009\end{array}$ \\
\hline \multicolumn{5}{|c|}{ Northern Sydney/Central Coast Area Health Service } \\
\hline North Sydney & 56.3 & 129.6 & 0.8 & 2.1 \\
\hline Central Coast & 36.8 & 170.8 & 0.7 & 4.3 \\
\hline \multicolumn{5}{|c|}{ Sydney South West Area Health Service } \\
\hline Central Sydney & 60.8 & 98.0 & 0.7 & 2.7 \\
\hline South West Sydney & 44.5 & 95.9 & 4.1 & 15.5 \\
\hline \multicolumn{5}{|c|}{ South Eastern Sydney/lllawarra Area Health Service } \\
\hline South East Sydney & 75.8 & 127.6 & 1.0 & 2.5 \\
\hline Illawarra & 41.3 & 276.5 & 0.7 & 4.8 \\
\hline \multicolumn{5}{|c|}{ Sydney West Area Health Service } \\
\hline Wentworth & 65.0 & 246.7 & 1.4 & 6.3 \\
\hline Western Sydney & 68.9 & 167.2 & 1.7 & 3.8 \\
\hline \multicolumn{5}{|c|}{ Greater Western Area Health Service } \\
\hline Far West & 45.5 & 126.3 & 2.6 & 6.7 \\
\hline Mid West & 26.6 & 149.7 & 1.5 & 3.6 \\
\hline Macquarie & 144.1 & 228.4 & 3.2 & 5.3 \\
\hline \multicolumn{5}{|c|}{ Greater Southern Area Health Service } \\
\hline Greater Murray & 63.7 & 158.7 & 1.2 & 3.8 \\
\hline Southern & 54.1 & 132.6 & 0.3 & 0.6 \\
\hline \multicolumn{5}{|c|}{ Hunter/New England Area Health Service } \\
\hline Hunter & 62.7 & 145.7 & 0.8 & 3.2 \\
\hline New England & 45.6 & 85.9 & 2.1 & 4.1 \\
\hline \multicolumn{5}{|c|}{ North Coast Area Health Service } \\
\hline Mid North Coast & 22.8 & 127.5 & 1.6 & 5.0 \\
\hline Northern Rivers & 40.3 & 285.1 & 1.5 & 7.4 \\
\hline NSW & 56.6 & 152.1 & 2.4 & 7.5 \\
\hline
\end{tabular}

hospitalisation rate was highest among infants aged less than 1 year in all years studied, with an annual average of 151.6 per 100000 population. Hospitalisation rates for children aged 1-19 years were relatively low in the early part of this decade and remained low until the current epidemic in 2008-2009. The 2008-2009 hospitalisation rates for infants aged less than 1 year and children aged 1-4 years were 4.3 and 4.5-fold higher respectively than the previous 5-year average for each age group. In contrast to children, the hospitalisation rate among adults aged 60 years and over has steadily increased, rising from 1.1 per 100000 population in 1999 to a peak of 6.8 per 100000 population in 2009. The 2008-2009 hospitalisation rate for adults aged 60 years and over was 1.8-fold higher than the previous 5-year average for this age group.

\section{Vaccination status}

Of the 2340 cases aged $1-4$ years in 2008-2009, $230(10 \%)$ were not vaccinated, $30(1 \%)$ reported receiving one to two doses, $1666(71 \%)$ three doses, 65 (3\%) four dose and 349 $(15 \%)$ had unknown doses recorded.

\section{Area health service distribution}

An increase in pertussis notifications was reported during the second half of 2008. The outbreak was first reported from the Sydney West and North Coast Area Health Services (AHSs), but by the end of 2008 an increase was being reported from all AHSs in NSW.

Over the epidemic period, the average notification rate varied across geographical areas (from 86 per 100000 population in rural New England to 285 per 100000 population in Northern Rivers). As with other years, the highest age-specific rates were reported from cases aged less than 1 year across all areas. A similar pattern of variation was observed in the hospitalisation data, however those AHSs with the highest notification rates did not always have the highest hospitalisation rates (Table 1).

\section{Method of diagnosis}

During 2008 and 2009, there was a change in testing practices for the diagnosis of pertussis in NSW (Figure 6). Prior to 2008, serology was the most common method of diagnosis 


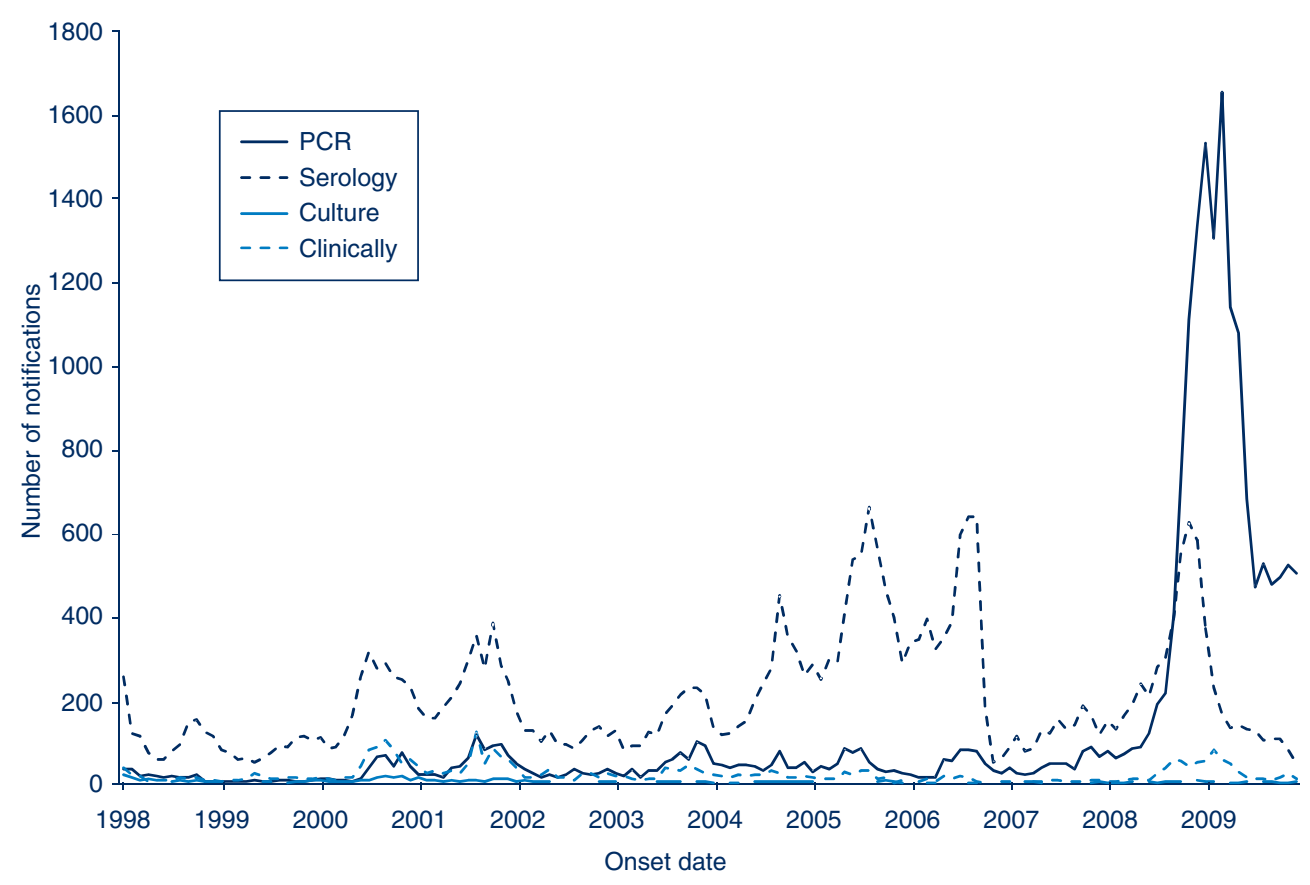

Figure 6. Pertussis notifications by clinical diagnosis compared with three methods of laboratory testing, NSW, 1998-2009.

Source: NSW Notifiable Diseases Database, NSW Department of Health.

in NSW, accounting for $85 \%$ of notifications between 2005 and 2007 ( $92 \%$ for persons aged 20 years and over). Over the epidemic period $26 \%$ of notifications were diagnosed by serology ( $78 \%$ of these in persons aged 20 years and over).

For children aged $0-5$ years, identification by polymerase chain reaction (PCR) increased from $67 \%$ of notifications in $2005-2007$ to $90 \%$ in $2008-2009$. The increase in notifications by PCR occurred across all ages in those aged $0-5$ years but was greatest for those aged 3 years (from $44 \%$ in $2005-$ 2007 to $87 \%$ notifications in 2008-2009).

\section{Severe morbidity and mortality}

In 2008 two deaths were reported in adults, and in 2009 one death was reported in an infant. During the 2008-2009 epidemic period, the overall median length of stay per hospital admission was 3 days. The median length of stay was longest in infants and elderly people (Table 2). For infants aged less than 1 year, the median length of stay decreased with increasing age. Intensive care unit admissions were more common in very young infants and elderly people (Table 2). One in 13 infants less than 2 months of age hospitalised with pertussis required an intensive care unit admission during their stay.

\section{Discussion}

Pertussis notifications increased significantly in 2008 and 2009 and were 2.7 times higher than the previous 5-year average in NSW. While the increase was initially reported from two AHSs (Sydney West and North Coast), all AHSs reported similar increases, with a peak in notifications during December 2008 and January 2009. Similarly, the hospitalisation rate for pertussis was 3.9 times higher than the previous 5-year average. A slight increase was also reported in hospitalised cases aged more than 60 years. As with notification data, hospitalisation rates during the epidemic period varied by age group with high rates reported from children aged 1-4 years. This finding was in contrast to previous outbreak years.

Notifications for those aged $10-14$ and 15-19 years increased during 2008-2009, although not to the extent reported in other age groups. In previous years, following the introduction of the adolescent booster dose in 2004, notifications for those aged 10-14 and 15-19 years declined. The increase in notifications for these age groups during 2008-2009 may be the result of a proportion of children in this age group who did not receive the booster dose; this is the subject of further investigation.

As with previous outbreak years, the highest age-specific rates were reported from infants aged less than 1 year. Notification rates also increased in children aged 1-4 and 5-9 years during the 2008-2009 epidemic, and was striking for children aged 3-4 years. For children aged $0-5$ years the biggest change in notifications was from those aged $0-11$ months (from $48 \%$ of notifications in $2005-2007$ to $25 \%$ in 2008-2009) and 3 years (from 14\% of notifications in $2005-2007$ to $25 \%$ in 2008-2009). The increase in notifications in children aged 3 years may be associated with waning immunity between the primary three doses and first booster as a result of changes in vaccination policy and removal of the 18-month booster in 2003.

When comparing the relative size of the 2008-2009 epidemic with previous epidemics, some caution must be applied as the 
Table 2. Number of hospitalisations, admissions to ICU and median length of hospital stay for pertussis, by age group, NSW, 2008-2009

\begin{tabular}{|c|c|c|c|c|}
\hline \multirow[t]{2}{*}{ Age group } & \multirow{2}{*}{$\begin{array}{l}\text { Hospital admissions } \\
n\end{array}$} & \multirow{2}{*}{$\begin{array}{c}\text { Median length of stay (days) } \\
n\end{array}$} & \multicolumn{2}{|c|}{ ICU admission } \\
\hline & & & $n$ & $\%$ total cases \\
\hline $0-1$ month & 207 & 4 & 16 & 8 \\
\hline 2-3 months & 251 & 3 & 15 & 6 \\
\hline 4-5 months & 87 & 2 & 3 & 3 \\
\hline 6-11 months & 58 & 2 & 4 & 7 \\
\hline $1-4$ years & 123 & 2 & 3 & 2 \\
\hline $5-9$ years & 29 & 1 & 0 & - \\
\hline $10-19$ years & 35 & 2 & 1 & 3 \\
\hline 20-59 years & 124 & 2 & 2 & 2 \\
\hline 60 years and over & 138 & 6 & 6 & 4 \\
\hline All ages & 1052 & 3 & 50 & 5 \\
\hline
\end{tabular}

apparent size of the 2008-2009 epidemic may also have been in part due to the use of more sensitive diagnostic tests. A shift to PCR testing, which is considered more sensitive than serology early in the illness, enabled the identification of cases that may otherwise have been undiagnosed. The increase in PCR testing occurred across all age groups and was greatest among adults aged 20 years and above $(8 \%$ in 2005-2007 compared to 50\% in 2008-2009). Among cases aged $0-5$ years, the greatest increase was noted for children aged 3 years. Further analysis of notification data for this age group will need to be undertaken to assess the possible effects of changes in immunisation policy in NSW.

To help protect infants and children during the epidemic, NSW Health advised parents and general practitioners that the first dose of DTPa vaccine (usually given at 2 months) could be given as early as 6 weeks of age and that the scheduled fourth dose (usually given at 4 years) could be administered from the age of 3 years and 6 months, to provide earlier protection against pertussis. ${ }^{11}$ In addition, NSW Health provided free (dTpa) vaccine for all new parents, grandparents and other adults who regularly care for infants aged less than 1 year.

\section{Conclusion}

This review of pertussis notifications and hospitalisations during the 2008-2009 epidemic identified epidemiological differences compared to previous outbreak years. The age distribution of cases, specifically those aged $0-4$ years, will require further investigation to determine the impact of changes to immunisation policy on pertussis notifications. The range of public health control measures that were implemented in NSW in response to the epidemic will require further evaluation.

\section{References}

1. WHO. Pertussis vaccines. WHO Position Paper. Wkly Epidemiol $\operatorname{Rec} 2005 ; 80(4): 31-9$
2. Wendelboe AM, Njamkepo E, Bourillon A, Floret D, Gaudelus J, Gerber M. Transmission of Bordetella pertussis to young infants. Pediatr Infect Dis J 2007; 26(4): 293-9. doi:10.1097/ 01.inf.0000258699.64164.6d

3. Quinn HE, McIntyre PB. Pertussis epidemiology in Australia over the decade 1995-2005-trends by region and age group. Commun Dis Intell 2007; 31(2): 205-15.

4. Salmaso S, Mastrantonio P, Tozzi AE, Stefanelli P, Anemona A, Ciofi degli Atti ML et al. Sustained efficacy during the first 6 years of life of 3-component acellular pertussis vaccines administered in infancy: the Italian experience. Pediatrics 2001; 108(5): E81. doi:10.1542/peds.108.5.e81

5. Torvaldsen S, McIntyre PB. Effect of the preschool pertussis booster on national notifications of disease in Australia. Pediatr Infect Dis J 2003; 22(11): 956-9. doi:10.1097/01.inf. 0000095198.75170.b6

6. Australian Bureau of Statistics. Australian Bureau of Statistics: Children's immunisation Australia (Catalogue No. 4352.0). Canberra: AGPS; 1995.

7. Hull B, Deeks S, Menzies R, McIntyre P. Immunisation coverage annual report, 2007. Commun Dis Intell 2009; 33(2): 170-87.

8. Brotherton J, McAnulty J. A pertussis epidemic in NSW: how epidemiology reflects vaccination policy. NS W Public Health Bull 2003; 14(4-5): 77-81. doi:10.1071/NB03024

9. Viney KA, McAnulty JM, Campbell-Lloyd S. Pertussis in NSW, 1993-2005: the impact of vaccination policy on pertussis epidemiology. N S W Public Health Bull 2007; 18(3-4); 55-61. doi:10.1071/NB07068

10. NSW Department of Health. Response protocol for NSW Public Health Units: pertussis. Available from: http://www.health.nsw. gov.au/factsheets/guideline/pertusis.html (Cited $30 \mathrm{March}$ 2010.)

11. Australian Technical Group on Immunisation (ATAGI). 41st Meeting: 15-16 October 2009, summary of outcomes. Available from: http://immunise.health.gov.au/internet/immunise/publish ing.nsf/Content/E7E989916C4FCAD8CA2576BF007D6B6E/ \$File/ATAGI-41-bulletin.pdf (Cited 30 March 2010.) 